\title{
Investigation of the Error Performance of Tunstall Coding
}

\author{
Johann A. Briffa \\ Dept. Comms. \& Computer Eng. \\ University of Malta \\ Msida MSD 06, Malta \\ E-mail: jabrif@eng.um.edu.mt
}

\author{
Victor Buttigieg \\ Dept. Comms. \& Computer Eng. \\ University of Malta \\ Msida MSD 06, Malta \\ E-mail: vjbutt@eng.um.edu.mt
}

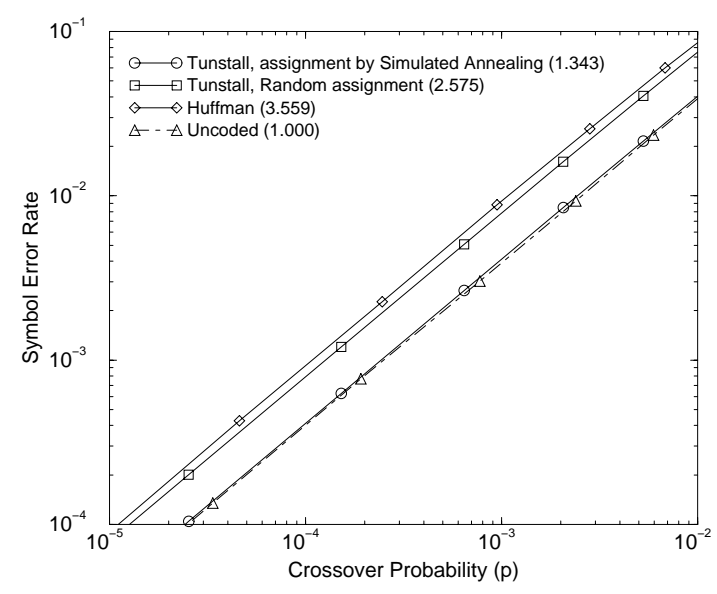

Fig. 1: Tunstall code performance ( $E_{s}$ given in parentheses)

Tunstall code is marginally better than that of the Huffman code. This was expected because Tunstall coding does not suffer from loss of synchronisation of codewords. Furthermore, when the Tunstall code is optimised, its error performance approaches that of the uncompressed stream.

\section{CONCLUSIONS}

since the Tunstall code is complete. This means that the sequence of source symbols represented by the original codeword will be replaced by the sequence corresponding to the incorrectly interpreted codeword. Now, the two sequences may not even be of the same length, leading to insertion, deletion as well as substitution errors in the decoded message. The Levenshtein distance [3], is used to calculate the number of symbol errors in the decoded message.

\section{Optimising the Error Performance}

The Error Span $\left(E_{s}\right)$ is defined as the average number of symbol errors in the decoded message for a random single bit error in the encoded message. Therefore, $E_{s}$ depends on the assignment of codewords to the sequences of source symbols. More importantly, this assignment will not affect the compression performance of the code. This feature has been exploited by seeking to assign codewords in such a way as to reduce $E_{s}$ (by designing a codebook such that random single-bit inversions in codewords result in another codeword mapping to a source symbol sequence very similar to the original one). Various code assignment algorithms have been investigated [4], representing different trade-offs between algorithm complexity and code performance.

To quantify the effect of $E_{s}$ on the code's performance in a noisy channel, the Symbol Error Rate (SER) of the decoded stream is plotted against the channel's Bit Error Rate (BER), as in Fig. 1. For comparison, the source was also encoded using a Huffman code. The performance of the randomly-assigned
The algorithms which obtain the best $E_{s}$ tend to be very resource intensive. The fast algorithms, while performing significantly better than the random assignment, are much less effective in reducing $E_{s}$. In practice, the simple algorithms are the only ones that can be used in adaptive compression, and in any other case where the source statistics are not fixed. In certain cases, however, an approximation to the actual source statistics is known, and the complexity of the codeword assignment algorithm is of no consequence. This makes it feasible to seek the best error performance possible.

By increasing the costs of insertion and deletion errors, a code can be designed which trades off substitutional error performance for a better synchronisation. This may be useful in image compression applications, where insertion and deletion are typically more visible than substitution errors.

\section{REFERENCES}

[1] B. P. Tunstall, Synthesis of Noiseless Compression Codes, Ph.D. thesis, Georgia Institute of Technology, 1968.

[2] James C. Maxted and John P. Robinson, "Error recovery for variable length codes," IEEE Transactions on Information Theory, vol. 31, no. 6, pp. 794-801, Nov. 1985.

[3] Joseph B. Kruskal, "An overview of sequence comparison: Time warps, string edits, and macromolecules," SIAM Review, vol. 25, no. 2, pp. 201-237, Apr. 1983.

[4] Johann A. Briffa, Investigation of the Error Performance of Tunstall Coding, B.Eng.(Hons.) Final Year Dissertation, University of Malta, Faculty of Engineering, 1997. 